\title{
Potential Benefits of Prophylactic Cholecystectomy in Patients Undergoing Bariatric Bypass Surgery
}

\author{
A. Hussain ${ }^{1}$ - S. EL-Hasani ${ }^{2}$
}

Published online: 2 February 2016

(C) Springer Science+Business Media New York 2016

Dear Editor,

We read with interest the article of Amstutz et al. [1] and congratulate the authors for selecting such as a practical and important subject. Basically, gallbladder/gallstone problem is associated with successful weight loss following bariatric surgery.

Performing laparoscopic cholecystectomy on morbidly/ super obese patients can be difficult, and this difficulty may be accentuated if the gallbladder is thickened, fibrosed and adherent to the surrounding tissue. This situation will increase the operative time and may lead to an increased morbidity. The large study of Worni et al. has clearly demonstrated increased morbidity and mortality as well as hospital stay if combined cholecystectomy and bariatric surgery is performed [2].

On the other hand, endoscopic assessment to the common bile duct following gastric bypass is not possible unless complex combined laparoscopic and endoscopic technique is applied.

The number of colleagues who are trained in laparoscopic exploration of the common bile duct and have the opportunity to do a large number of patients is diminishing.

The cost effectiveness of the combined approach versus post bariatric surgery cholecystectomy is to be balanced against longer hospital stay, increased morbidity and mortality as per the study of Worni et al.

A. Hussain

azahrahussain@yahoo.com

1 Brighton and Sussex University Hospital, Brighton, UK

2 Kings College Hospital, London, UK
In our unit and after experience of thousands of weight loss procedures, we have adopted the following protocol that we thought we should share it with the bariatric community to compliment the scientific value of this excellent article:

1. Patients who have symptomatic gallstones will have laparoscopic cholecystectomy before any bariatric procedure.

2. Patients who have asymptomatic gall stones will go ahead with their bariatric procedures and then offered laparoscopic cholecystectomy 6 months afterwards when they have had a significant amount of weight loss.

3. Patients who do not have gallstones and underwent successful weight loss surgery are offered ultrasound scan of the gallbladder a year after surgery, and if the ultrasound scan confirms gallstones, we will offer them laparoscopic cholecystectomy even if they are asymptomatic.

Compliance with Ethical Standards Does not apply to this article

Informed Consent Does not apply to this article

Conflict of Interest The authors declare that they have no competing interests.

\section{References}

1. Amstutz S, Michel J-M, Kopp S, et al. Potential benefits of prophylactic cholecystectomy in patients undergoing bariatric bypass surgery. Obes Surg. 2015;25(11):2054-60.

2. Worni M, Guller U, Shah A, et al. Cholecystectomy concomitant with laparoscopic gastric bypass: a trend analysis of the nationwide inpatient sample from 2001 to 2008. Obes Surg. 2012;22(2):220-9. 